\title{
(Sub)millimeter emission lines of molecules in born-again stars ${ }^{\star}$
}

\author{
D. Tafoya ${ }^{1}$, J. A. Toalá ${ }^{2}$, W. H. T. Vlemmings ${ }^{1}$, M. A. Guerrero ${ }^{3}$, E. De Beck ${ }^{1}$, M. González ${ }^{3}$, S. Kimeswenger ${ }^{4,5}$, \\ A. A. Zijlstra ${ }^{6}$, Á. Sánchez-Monge ${ }^{7}$, and S. P. Treviño-Morales ${ }^{8,9}$
}

\author{
${ }^{1}$ Chalmers University of Technology, Onsala Space Observatory, 43992 Onsala, Sweden \\ e-mail: daniel.tafoya@chalmers.se \\ 2 Institute of Astronomy and Astrophysics, Academia Sinica (ASIAA), 10617 Taipei, Taiwan, ROC \\ 3 Instituto de Astrofísica de Andalucía (IAA-CSIC), Glorieta de la Astronomía s/n, 18008 Granada, Spain \\ 4 Instituto de Astronomía, Universidad Católica del Norte, Av. Angamos 0610, Casilla 1280 Antofagasta, Chile \\ 5 Institut für Astro- und Teilchenphysik, Universität Innsbruck, Technikerstr. 25/8, 6020 Innsbruck, Austria \\ 6 Jodrell Bank Centre for Astrophysics, School of Physics and Astronomy, University of Manchester, Manchester M13 9PL, UK \\ 7 I. Physikalisches Institut, Universität zu Köln, Zülpicher Str. 77, 50937 Cologne, Germany \\ 8 Instituto de Ciencia de Materiales de Madrid, Sor Juana Inés de la Cruz 3, 28049 Cantoblanco, Madrid, Spain \\ 9 Observatorio Astronómico Nacional, Apdo. 112, 28803 Alcalá de Henares Madrid, Spain
}

Received 16 November 2016 / Accepted 20 December 2016

\begin{abstract}
Context. Born-again stars provide a unique possibility to study the evolution of the circumstellar envelope of evolved stars in human timescales. Up until now, most of the observations of the circumstellar material in these stars have been limited to studying the relatively hot gas and dust. In other evolved stars, the emission from rotational transitions of molecules, such as CO, is commonly used to study the cool component of their circumstellar envelopes. Thus, the detection and study of molecular gas in born-again stars is of great importance when attempting to understand their composition and chemical evolution. In addition, the molecular emission is an invaluable tool for exploring the physical conditions, kinematics, and formation of asymmetric structures in the circumstellar envelopes of these evolved stars. However, up until now, all attempts to detect molecular emission from the cool material around born-again stars have failed.

Aims. We searched for emission from rotational transitions of molecules in the hydrogen-deficient circumstellar envelopes of bornagain stars to explore the chemical composition, kinematics, and physical parameters of the relatively cool gas.

Methods. We carried out observations using the APEX and IRAM $30 \mathrm{~m}$ telescopes to search for molecular emission toward four well-studied born-again stars, V4334 Sgr, V605 Aql, A30, and A78, that are thought to represent an evolutionary sequence.

Results. For the first time, we detected emission from $\mathrm{HCN}$ and $\mathrm{H}^{13} \mathrm{CN}$ molecules toward V4334 Sgr, and CO emission in V605 Aql. No molecular emission was detected above the noise level toward A30 and A78. The detected lines exhibit broad linewidths $\gtrsim 150 \mathrm{~km} \mathrm{~s}^{-1}$, which indicates that the emission comes from gas ejected during the born-again event, rather than from the old planetary nebula. A first estimate of the $\mathrm{H}^{12} \mathrm{CN} / \mathrm{H}^{13} \mathrm{CN}$ abundance ratio in the circumstellar environment of $\mathrm{V} 4334 \mathrm{Sgr}$ is $\approx 3$, which is similar to the value of the ${ }^{12} \mathrm{C} /{ }^{13} \mathrm{C}$ ratio measured from other observations. We derived a rotational temperature of $T_{\text {rot }}=13 \pm 1 \mathrm{~K}$, and a total column density of $N_{\mathrm{HCN}}=1.6 \pm 0.1 \times 10^{16} \mathrm{~cm}^{-2}$ for V4334 Sgr. This result sets a lower limit on the amount of hydrogen that was ejected into the wind during the born-again event of this source. For V605 Aql, we obtained a lower limit for the integrated line intensities $I_{12} \mathrm{CO} / I_{13} \mathrm{CO}>4$.
\end{abstract}

Key words. stars: AGB and post-AGB - stars: mass-loss - circumstellar matter - stars: carbon - planetary nebulae: general

\section{Introduction}

The term born-again star is commonly used to refer to a postasymptotic giant branch (post-AGB) star that temporarily revisits the AGB as a consequence of experiencing a final helium shell flash (Iben 1984). During this phase, the born-again star undergoes a sudden episode of intense mass-loss in the form of a stellar wind, resulting in the formation of a hydrogenpoor (H-poor) thick circumstellar envelope (CSE) that enshrouds the star and renders it invisible at optical wavelengths (e.g. Duerbeck et al. 2000). After most of the gas on top of the stellar core is blown away by the aforementioned stellar wind, the

* This publication is based on data acquired with the Atacama Pathfinder Experiment (APEX) and IRAM $30 \mathrm{~m}$ telescopes. APEX is a collaboration between the Max-Planck-Institut für Radioastronomie, the European Southern Observatory, and the Onsala Space Observatory. IRAM is supported by INSU/CNRS (France), MPG (Germany) and IGN (Spain). surface temperature begins to rise and a tenuous fast stellar wind takes over. At this point the star begins to re-trace the postAGB track, while still burning helium on a thin layer around its core. In a similar way to the interacting stellar winds scenario for the creation of a planetary nebula (PN, plural $\mathrm{PNe}$ ), the fast wind sweeps up the recently formed H-poor CSE and creates a compressed shell (Kwok et al. 1978). Once the star becomes hot enough $\left(T_{\text {eff }} \gtrsim 30000 \mathrm{~K}\right)$, the energetic stellar radiation ionizes the shell and a brand new PN forms (sometimes referred to as "born-again PN"; e.g. Guerrero et al. 2012). If the final helium shell flash occurs when the star is already on the white dwarf cooling track, which is known as a very late thermal pulse (VLTP), it is possible that the born-again PN becomes visible while the old PN is still detectable. This shows up in the images as a bright compact PN nested inside a faint extended PN (e.g. Jacoby 1979). Observations of stars that are undergoing the born-again event have shown that the evolution from the bornagain AGB star to the formation of a born-again PN happens 
on a surprisingly rapid timescale of tens of years. This implies that these objects offer us the unique possibility of studying the transformation of an AGB star into a PN on human timescales.

There is a small group of sources that are thought to have experienced a VLTP in relatively recent epochs and that currently are at different stages of their post-final flash evolution (Zijlstra 2002). The most recent born-again event was observed in the late 1990s toward the central star of a planetary nebula (CSPN) located in the constellation of Sagittarius (Nakano et al. 1996; Benetti et al. 1996; Duerbeck et al. 1996). The object was cataloged with the variable star name V4334 Sgr, but it is commonly known as Sakurai's object, since it was discovered by the amateur astronomer Y. Sakurai. Another object that is thought to have experienced a born-again event in a very similar way to Sakurai's object, but 75 years earlier, is the star cataloged as V605 Aql, which is the central star of the planetary nebula A58 (PN A66 58; PN G037.505.1) (Wolf 1920; Seitter 1987; Guerrero \& Manchado 1996; Clayton \& De Marco 1997). At present, this star exhibits spectral characteristics of a Wolf-Rayet [WC] CSPN with a temperature of $\sim 100 \mathrm{kK}$ and its H-poor CSE has been partially photoionized (Clayton et al. 2006; van Hoof et al. 2007). Finally, two post-VLTP objects that have already developed a born-again PN from the hydrogen-deficient material that was ejected during the born-again AGB phase are the central stars of the PNe A30 (PN A66 30; PN G208.5+33.2) and A78 (PN A66 78; PN G081.214.9) (Guerrero et al. 2012; Toalá et al. 2015). It has been estimated that these sources experienced a born-again event a thousand years ago (Fang et al. 2014).

The four objects described above are often considered in the literature to represent different stages of an evolutionary sequence of born-again stars. Moreover, a striking common characteristic of all these objects is that the circumstellar material ejected in the born-again event seems to be distributed in an equatorial disk-like structure and a bipolar outflow (Borkowski et al. 1993; Guerrero \& Manchado 1996; Chu et al. 1997; Hinkle et al. 2008; Chesneau et al. 2009; Hinkle \& Joyce 2014). This characteristic is particularly interesting because explaining the formation of bipolar structures in evolved stars represents one of the major current challenges in stellar evolution (Balick \& Frank 2002; De Marco 2014). Thus, these objects, which are developing bipolar morphologies in real time are ideal for studying the formation of asymmetric PNe. Extensive theoretical work has been devoted to explain the evolution of born-again stars (e.g. Herwig 2002; Miller Bertolami et al. 2006; Fang et al. 2014; Woodward et al. 2015, and references therein). In addition, these sources have been observed over a broad range of wavelengths, from X-rays to radio waves (e.g. Hajduk et al. 2005; Toalá et al. 2015, and references therein). However, to date the detection of molecular emission at millimeter and submillimeter wavelengths, which traces the cooler molecular component of the CSE, has been elusive. In this paper we present observations carried out with the $12 \mathrm{~m}$ Atacama Pathfinder Experiment (APEX) and the $30 \mathrm{~m}$ Institute for Radio Astronomy in the Millimeter Range (IRAM 30 m) telescopes. We report on the first detection of molecular emission lines toward born-again stars. This result opens a new window to study in real time the evolution of the physical and chemical properties of the hitherto unexplored cool gas around born-again AGB stars.

\section{Observations}

The observations with APEX were carried out in July and November 2013, and March 2016, toward the sources V4334 Sgr and V605 Aql. The typical precipitable water vapor level during the observations was $\sim 1 \mathrm{~mm}$ and the total on-source integration time was about 1.7 and $5.5 \mathrm{~h}$ for V4334 Sgr and V605 Aql, respectively. The heterodyne receiver APEX-2, which covers the frequency range from 267 to $378 \mathrm{GHz}$, was used as a frontend. In the backend, we used the XFFTS spectrometer, which consists of two units that provide an instantaneous bandwidth of $2.5 \mathrm{GHz}$ and 32768 spectral channels each. There is a fixed overlap region of $1.0 \mathrm{GHz}$ between the units, hence the effective bandwidth was $4.0 \mathrm{GHz}$ centered at the rest frequency of the $\mathrm{CO}(J=3 \rightarrow 2)$ and $\operatorname{HCN}(J=4 \rightarrow 3)$ transitions.

The observations with the IRAM $30 \mathrm{~m}$ telescope were carried out in May and June 2014. The frontend was the Eight MIxer Receiver (EMIR), which covers four bands in the frequency range from $\sim 80$ to $360 \mathrm{GHz}$. The Fast Fourier Transform Spectrometer (FTS) was used as a backend. The observations were done in the EMIR bands E090 and E230, covering the frequency ranges $84-116 \mathrm{GHz}$ and $228.5-240.5 \mathrm{GHz}$, respectively, with a spectral resolution of $200 \mathrm{kHz}$. The setup enabled us to simultaneously look for emission lines of, for example, $\mathrm{CO},{ }^{13} \mathrm{CO}$, and HCN. The observations with this telescope included scans toward the four born-again stars, V4334 Sgr, V605 Aql, A30, and A78. However, owing to an error in the pointing of the observations on V605 Aql, we had to exclude these data from this work.

The temperature scale of the data that was delivered by the observatories was in units of the antenna temperature corrected for the atmospheric attenuation, $T_{\mathrm{A}}^{\prime}$. To compare our observations with other results, the antenna temperature was converted to main-beam brightness temperature, $T_{\mathrm{MB}}$, and to flux density, $S_{v}$. The main-beam brightness temperature is obtained as $T_{\mathrm{MB}}=T_{\mathrm{A}}^{\prime} / \eta_{\mathrm{MB}}$, where $\eta_{\mathrm{MB}}$ is the main beam efficiency. The relation between antenna temperature and flux density is expressed as $S_{v}=T_{\mathrm{A}}^{\prime} / \Gamma$, where $\Gamma$ is the point-source sensitivity of the antenna. The sensitivity can be calculated as $\left[\Gamma / \mathrm{K} \mathrm{Jy}^{-1}\right]=$ $\left(\eta_{\mathrm{A}}[D / \mathrm{m}]^{2}\right) / 3520$, where $D$ is the diameter of the telescope and $\eta_{\mathrm{A}}$ is the aperture efficiency (Wilson et al. 2013). A summary of the observation parameters is presented in Table 1 . The software CLASS of GILDAS ${ }^{1}$ was used to average the spectral scans, remove baselines and smooth the spectral resolution. All the spectra were re-sampled to a spectral resolution of $20 \mathrm{~km} \mathrm{~s}^{-1}$.

\section{Results}

From our observations we detected, for the first time, molecular line emission toward two of the targeted born-again stars. Emission from the molecule $\mathrm{HCN}$ and its isotopologue $\mathrm{H}^{13} \mathrm{CN}$ was found in V4334 Sgr. On the other hand, $\mathrm{CO}(J=3 \rightarrow 2)$ emission was detected toward V605 Aql. Although the lines are relatively weak, our deep observations revealed emission of these molecules over a broad range of velocities with a $S / N \gtrsim 3$ for the line peaks (see Figs. 1-3). We fitted Gaussian profiles to the detected lines to obtain their relevant parameters. The best-fit values for the peak-temperature, $T_{\mathrm{MB} \text {,peak }}$, central velocity, $v_{\mathrm{LSR}}$, and linewidths, $\Delta v$, are listed in Table 2 . Table 3 shows the channelto-channel $\mathrm{rms}$ noise level of the spectra where no molecular emission was detected. In the following we describe the results of the observations for each source.

1 See http://www.iram.fr/IRAMFR/GILDAS for more information about the GILDAS softwares. 
Table 1. Parameters of the observations.

\begin{tabular}{|c|c|c|c|c|c|c|c|}
\hline $\begin{array}{l}\text { Source } \\
\text { (name) }\end{array}$ & $\begin{array}{l}\text { Equatorial } \\
\text { (h m s }\end{array}$ & $\begin{array}{c}\text { coordinates } \\
\circ, \prime \prime)\end{array}$ & $\begin{array}{l}\text { Frequency range } \\
(\mathrm{GHz})\end{array}$ & $\begin{array}{c}\text { Beam size }^{a} \\
(I \prime)\end{array}$ & $\eta_{\mathrm{MB}}^{b}$ & $\eta_{\mathrm{A}}^{c}$ & $\begin{array}{c}(1 / \Gamma)^{d} \\
\left(\mathrm{Jy} \mathrm{K}^{-1}\right)\end{array}$ \\
\hline \multicolumn{8}{|c|}{ APEX } \\
\hline \multirow[t]{2}{*}{ V4334 Sgr } & 175232.69 & -174108.0 & $352.5-356.5$ & $17.3-17.1$ & 0.73 & 0.60 & 40.7 \\
\hline & & & $343.8-347.8$ & $17.8-17.6$ & 0.73 & 0.60 & 40.7 \\
\hline \multirow[t]{2}{*}{ V605 Aql } & 191820.47 & 014659.6 & $343.8-347.8$ & $17.8-17.6$ & 0.73 & 0.60 & 40.7 \\
\hline & & & $328.5-332.5$ & $18.6-18.3$ & 0.73 & 0.60 & 40.7 \\
\hline \multicolumn{8}{|c|}{ IRAM $30 \mathrm{~m}$} \\
\hline \multirow[t]{3}{*}{ V4334 Sgr } & 175232.69 & -174108.0 & $228.5-236.5$ & $10.8-10.4$ & 0.59 & 0.46 & 8.5 \\
\hline & & & $100.5-116.5$ & $24.5-21.1$ & 0.78 & 0.62 & 6.4 \\
\hline & & & $84.5-100.5$ & $29.1-24.5$ & 0.81 & 0.63 & 6.2 \\
\hline \multirow[t]{3}{*}{ A 30} & 084653.49 & 175246.8 & $228.5-236.5$ & $10.8-10.4$ & 0.59 & 0.46 & 8.5 \\
\hline & & & $100.5-116.5$ & $24.5-21.1$ & 0.78 & 0.62 & 6.4 \\
\hline & & & $84.5-100.5$ & $29.1-24.5$ & 0.81 & 0.63 & 6.2 \\
\hline \multirow[t]{3}{*}{ A78 } & 213529.38 & 314145.3 & $228.5-236.5$ & $10.8-10.4$ & 0.59 & 0.46 & 8.5 \\
\hline & & & $100.5-116.5$ & $24.5-21.1$ & 0.78 & 0.62 & 6.4 \\
\hline & & & $84.5-100.5$ & $29.1-24.5$ & 0.81 & 0.63 & 6.2 \\
\hline
\end{tabular}

Notes. ${ }^{(a)}$ The fullwidth at half maximum $(\mathrm{FWHM})$ of the telescope primary beam can be computed approximately as $F W H M_{\mathrm{APEX}}=17$ !' $^{\prime} \times$ $\left(345 /\left[v_{\mathrm{obs}} / \mathrm{GHz}\right]\right)$, and $F W H M_{\text {IRAM }}=10 . \prime 7 \times\left(230 /\left[v_{\mathrm{obs}} / \mathrm{GHz}\right]\right)$, where $v_{\mathrm{obs}}$ is the observation frequency. ${ }^{(b)}$ Main beam efficiency of the telescope. The main-beam brightness temperature is obtained as $T_{\mathrm{MB}}=T_{\mathrm{A}}^{\prime} / \eta_{\mathrm{MB}} .{ }^{(c)}$ Apperture efficiency of the telescope. ${ }^{(d)}$ Inverse of the point source sensitivity of the telescope. The relation between antenna temperature and flux density is expressed as $S_{v}=T_{\mathrm{A}}^{\prime} / \Gamma$.
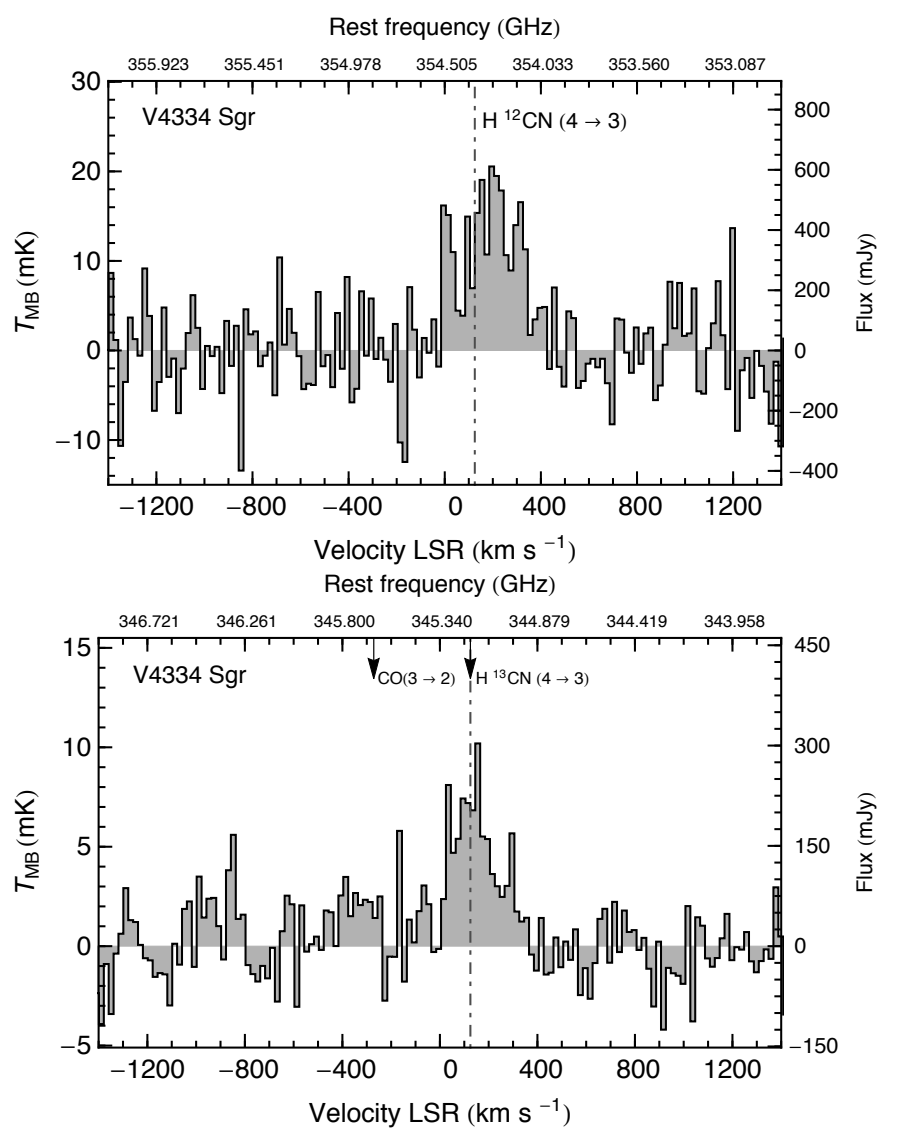

Fig. 1. V4334 Sgr spectra of the emission line detected with APEX. The width of the channels is $20 \mathrm{~km} \mathrm{~s}^{-1}$ and the dotted vertical line corresponds to the systemic velocity of the source, $v_{\mathrm{sys}}=125 \mathrm{~km} \mathrm{~s}^{-1}$ (Duerbeck et al. 1997). The flux density in the right axis was calculated using the corresponding value of $\Gamma$ shown in Table 1. Top: spectrum of the $\operatorname{HCN}(J=4 \rightarrow 3)$ emission. Bottom: spectrum of the $\mathrm{H}^{13} \mathrm{CN}(J=4 \rightarrow 3)$ emission. The arrows in the upper axis indicate the rest frequencies for the $\mathrm{CO}(J=3 \rightarrow 2)$ and $\mathrm{H}^{13} \mathrm{CN}(J=4 \rightarrow 3)$ lines.

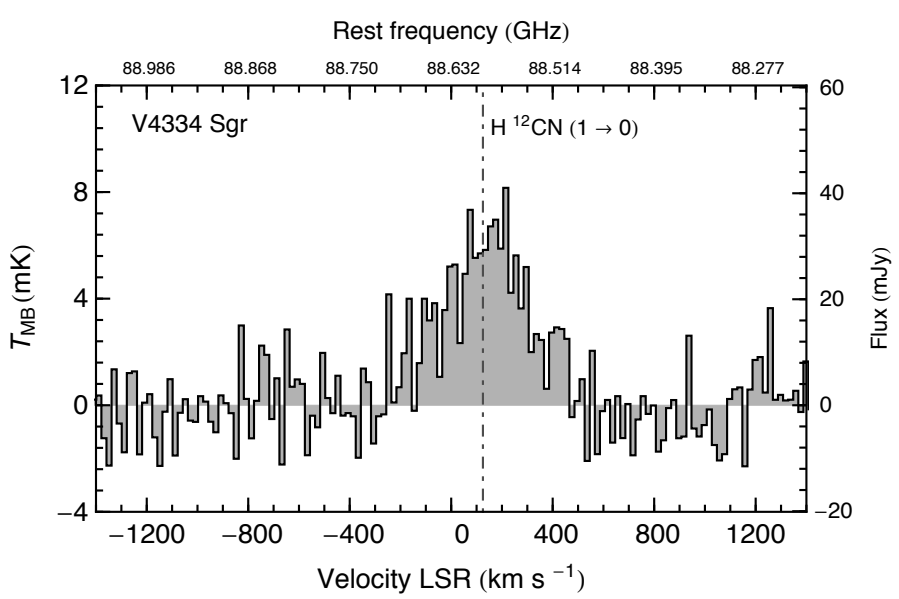

Fig. 2. IRAM $30 \mathrm{~m}$ spectrum of the $\operatorname{HCN}(J=1 \rightarrow 0)$ emission in V4334 Sgr (Sakurai's object). The width of each channel is $20 \mathrm{~km} \mathrm{~s}^{-1}$. The dotted vertical line corresponds to the systemic velocity of the source, $v_{\mathrm{sys}}=125 \mathrm{~km} \mathrm{~s}^{-1}$ (Duerbeck et al. 1997). The flux density in the right axis was calculated using the corresponding value of $\Gamma$ shown in Table 1.

\subsection{V4334 Sgr (Sakurai's object)}

Among the initial goals of the APEX observations was the detection of $\mathrm{CO}(J=3 \rightarrow 2)$ emission toward V4334 Sgr, but it was not detected above a rms noise level of $57 \mathrm{mJy}$ (Table 3). However, from these observations we serendipitously detected emission from the transition $J=4 \rightarrow 3$ of the isotopologue $\mathrm{H}^{13} \mathrm{CN}$ instead, which lies at a frequency $\approx 0.46 \mathrm{GHz}$ away from the one of the $\mathrm{CO}(J=3 \rightarrow 2)$ line (see lower panel of Fig. 1 and Table 2). Since the $\mathrm{H}^{13} \mathrm{CN}(J=4 \rightarrow 3)$ line was detected, we carried out additional observations with APEX to search for $\mathrm{HCN}(J=4 \rightarrow 3)$ emission with the aim of comparing their relative intensities. The $\mathrm{HCN}(J=4 \rightarrow 3)$ emission was successfully detected with a peak-temperature $\approx 2.3$ times higher than the one of its isotopologue $\mathrm{H}^{13} \mathrm{CN}$ (upper panel of Fig. 1). From the IRAM 30 m telescope observations, we also obtained 


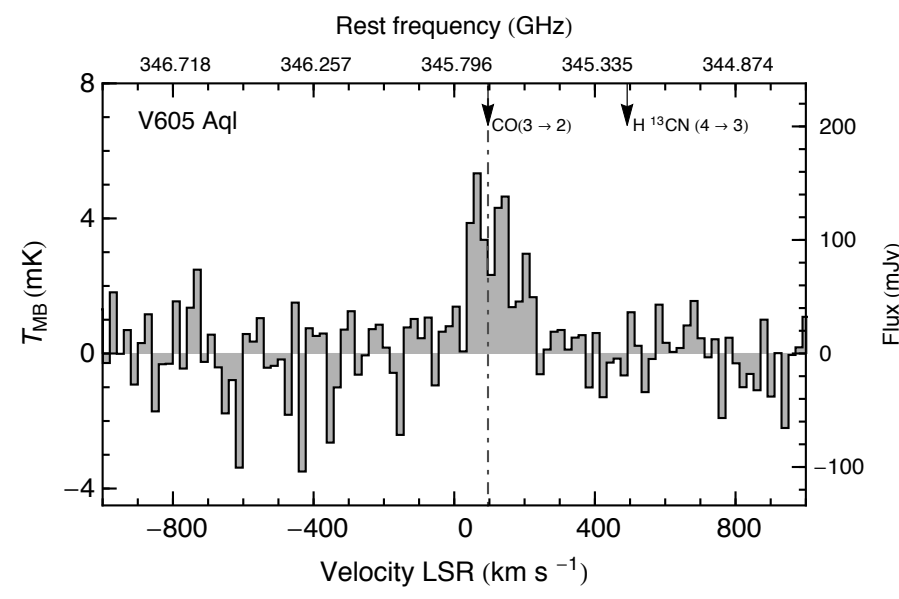

Fig. 3. APEX spectrum of the $\mathrm{CO}(J=3 \rightarrow 2)$ emission in V605 Aql. The width of each channel is $20 \mathrm{~km} \mathrm{~s}^{-1}$. The dotted vertical line corresponds to the systemic velocity relative to the local standard of rest of the PN A58, $v_{\mathrm{sys}}=96 \mathrm{~km} \mathrm{~s}^{-1}$, estimated by Clayton et al. (2013). The arrows indicate the rest frequencies for the $\operatorname{CO}(J=3 \rightarrow 2)$ and $\mathrm{H}^{13} \mathrm{CN}(J=4 \rightarrow 3)$ lines. The flux density in the right axis was calculated using the corresponding value of $\Gamma$, shown in Table 1 .

a clear detection of $\operatorname{HCN}(J=1 \rightarrow 0)$ emission at a frequency $\sim 88.6 \mathrm{GHz}$ (Fig. 2). We did not detect $\mathrm{CO}$ nor ${ }^{13} \mathrm{CO}$ emission above the rms noise level from these observations (see Table 3).

In general, the central velocities of the $\mathrm{HCN}$ and $\mathrm{H}^{13} \mathrm{CN}$ lines that were obtained from the Gaussian fits are in agreement with the systemic velocity of the source estimated from other observations, $v_{\text {sys }}=125 \mathrm{~km} \mathrm{~s}^{-1}$ (Duerbeck et al. 1997), which indicates that the emission is indeed associated with the source. We note that the central velocity of the $\operatorname{HCN}(J=4 \rightarrow 3)$ line differs from the systemic velocity of the source by $\approx 56 \mathrm{~km} \mathrm{~s}^{-1}$, which corresponds to a departure of $\sim 3.5 \sigma$ from the nominal value, given the standard deviation obtained from the Gaussian fit. However, since the other lines are consistent with the systemic velocity, it is likely that this discrepancy is due to limitations in the fit as a consequence of the lower $\mathrm{S} / \mathrm{N}$ of this line.

The widths of the lines show that the emitting material is expanding at a relatively large velocity $\left(v_{\exp }=\Delta v / 2 \gtrsim 100 \mathrm{~km} \mathrm{~s}^{-1}\right)$. Similar expansion velocities have been derived from the emission of optical lines seen in this source (e.g. Worters et al. 2009; Hinkle \& Joyce 2014). We notice that the widths of the $\mathrm{HCN}(J=4 \rightarrow 3)$ and $\mathrm{H}^{13} \mathrm{CN}(J=4 \rightarrow 3)$ lines seem to be narrower than the linewidth of the $\operatorname{HCN}(J=1 \rightarrow 0)$ emission (see Figs. 1 and 2, and Table 2). This could indicate that, in the region from where the $\mathrm{HCN}(J=1 \rightarrow 0)$ emission is arising, cooler gas (i.e. brighter at lower $J$ transitions) is probably associated with a faster component of the circumstellar envelope. Another possibility is that emission from faint, high-velocity, wings of the $J=4 \rightarrow 3$ transitions is not being detected owing to the higher rms noise of their spectra.

\subsection{V605 Aql}

Emission from $\mathrm{CO}(J=3 \rightarrow 2)$ was detected toward V605 Aql using the APEX telescope (see Fig. 3 and Table 2). The emission is centered at the velocity $v_{\mathrm{LSR}}=96 \pm 11 \mathrm{~km} \mathrm{~s}^{-1}$. The value is in excellent agreement with the systemic velocity derived by Clayton et al. (2013), $v_{\text {sys,LSR }}=96 \mathrm{~km} \mathrm{~s}^{-1}$, from modeling the broad emission of optical lines of [OIII] and [NII], which are attributed to the compact hydrogen-deficient nebula (Pollacco et al. 1992).
The expansion velocity of the molecular gas, as suggested by the width of the CO line of our observations, is $v_{\exp } \sim 80 \mathrm{~km} \mathrm{~s}^{-1}$. This is considerably lower than the value of $v_{\exp } \sim 215 \mathrm{~km} \mathrm{~s}^{-1}$ derived from the spectral modeling of the [OIII] and [NII] lines performed by Clayton et al. (2013). This indicates that the neutral (denser) gas, traced by the $\mathrm{CO}$ emission, expands at lower velocities than the ionized (less dense) material, which is in agreement with the results of numerical simulations of the formation of a born-again PN (Fang et al. 2014). No emission of the $\mathrm{H}^{13} \mathrm{CN}(J=4 \rightarrow 3)$ line was detected in V605 Aql above the noise level $1 \sigma$ of $29 \mathrm{mJy}$ (see Fig. 3 and Table 3 ).

\section{3. $A 30$ and $A 78$}

These sources were observed using only the IRAM $30 \mathrm{~m}$ telescope. We did not detect any line emission in the frequency bands of our observations. The channel-to-channel rms noise level of the spectra of these sources in the frequency ranges corresponding to rotational transitions of the molecules $\mathrm{CO},{ }^{13} \mathrm{CO}$, and $\mathrm{HCN}$ is shown in Table 3.

\section{Discussion}

\subsection{Molecular emission as a probe of the CSE of born-again stars}

To date, most of the studies on the morphology, kinematics, and chemical composition of CSEs of born-again-stars are based on observations at optical and infrared wavelengths (e.g. Hinkle \& Joyce 2014, and references therein). The observations reveal that, shortly after the star commences its journey back to the AGB track, its chemical composition exhibits dramatic changes (e.g. Eyres et al. 1998). This is followed by the condensation of molecular material and dust into a thick circumstellar shell that enshrouds the star, which renders it invisible at optical wavelengths. Studies of absorption features in the near and mid-infrared have revealed the presence of molecules such as $\mathrm{CO}, \mathrm{C}_{2}, \mathrm{CN}, \mathrm{HCN}$, among others in the CSE of the two youngest born-again stars (Clayton \& De Marco 1997; Eyres et al. 1998; Pavlenko et al. 2004; Evans et al. 2006). Infrared emission from dust in globules has also been observed toward A30 and A78 (Borkowski et al. 1994; Kimeswenger et al. 1998; Phillips \& Ramos-Larios 2007), but no molecular emission has been reported for these sources.

The emission of the $\mathrm{CO}$ molecule has proven to be an invaluable tool for studying the morphology and kinematics, as well as the physical conditions of the cool gas around a wide variety of evolved stars, from AGB stars to PNe. Previous searches of $\mathrm{CO}$ emission in born-again stars at millimeter and sub-millimeter wavelengths yielded inconclusive results and/or non-detections. Kameswara-Rao et al. (1991) reported a tentative $2.6 \sigma$ detection of $\mathrm{CO}(J=1 \rightarrow 0)$ emission using the Swedish-ESO Submillimetre Telescope (SEST) toward V605 Aql. The signal showed a peak main-beam temperature of $T_{\mathrm{MB}}=0.024 \mathrm{~K}(0.65 \mathrm{Jy})$ at the position of the LSR velocity of $138.5 \mathrm{~km} \mathrm{~s}^{-1}$. These authors concluded that this emission must originate in the old PN, based on the argument that the velocity of the hydrogen deficient nebula differs by $60 \mathrm{~km} \mathrm{~s}^{-1}$ from the LSR of the detected signal. However, given the relatively large expansion velocity of the molecular gas ejected during the born-again event, $v_{\exp } \sim 80 \mathrm{~km} \mathrm{~s}^{-1}$ (as obtained from our observations) $\mathrm{CO}(J=1 \rightarrow 0)$ emission from the hydrogen deficient nebula cannot be ruled out at such LSR velocity. Unfortunately, the spectrum of these observations was 
Table 2. Detected line emission.

\begin{tabular}{|c|c|c|c|c|c|c|c|c|}
\hline Molecule & Transition & $\begin{array}{l}\text { Rest frequency } \\
(\mathrm{GHz})\end{array}$ & $\begin{array}{c}\text { Line peak }\left(T_{\mathrm{MB}, \text { peak }}\right)^{a, b} \\
(\mathrm{mK})\end{array}$ & $\begin{array}{c}\text { Line peak }\left(S_{v, \text { peak }}\right)^{c} \\
(\mathrm{mJy})\end{array}$ & $\begin{array}{c}v_{\mathrm{LSR}}{ }^{a, d} \\
\left(\mathrm{~km} \mathrm{~s}^{-1}\right)\end{array}$ & $\begin{array}{c}\Delta v^{a, f} \\
\left(\mathrm{~km} \mathrm{~s}^{-1}\right)\end{array}$ & $\begin{array}{c}I^{f} \\
\left(\mathrm{~K} \mathrm{~km} \mathrm{~s}^{-1}\right)\end{array}$ & $\begin{array}{r}\mathrm{rms}^{c, g} \\
(\mathrm{mJy})\end{array}$ \\
\hline \multicolumn{9}{|c|}{ V4334 Sgr } \\
\hline $\mathrm{HCN}$ & $J=4 \rightarrow 3$ & 354.50548 & $16.5 \pm 1.8$ & $490 \pm 52$ & $181 \pm 16$ & $298 \pm 37$ & $4.9 \pm 0.8$ & 139 \\
\hline $\mathrm{HCN}$ & $J=1 \rightarrow 0$ & 88.63185 & $6.2 \pm 0.4$ & $31 \pm 2$ & $128 \pm 14$ & $405 \pm 34$ & $2.5 \pm 0.3$ & 7 \\
\hline $\mathrm{H}^{13} \mathrm{CN}$ & $J=4 \rightarrow 3$ & 345.33976 & $7.2 \pm 0.8$ & $215 \pm 24$ & $123 \pm 13$ & $236 \pm 30$ & $1.7 \pm 0.3$ & 57 \\
\hline \multicolumn{9}{|c|}{ V605 Aql } \\
\hline $\mathrm{CO}$ & $J=3 \rightarrow 2$ & 345.79599 & $4.0 \pm 0.5$ & $119 \pm 16$ & $96 \pm 11$ & $164 \pm 25$ & $0.7 \pm 0.1$ & 29 \\
\hline
\end{tabular}

Notes. ${ }^{(a)}$ Nominal value and standard error obtained from fitting a Gaussian model to the emission line. ${ }^{(b)}$ The main-beam brightness temperature was obtained as $T_{\mathrm{MB}}=T_{\mathrm{A}}^{\prime} / \eta_{\mathrm{MB}}$, where $T_{\mathrm{A}}^{\prime}$ is the antenna temperature corrected for the atmospheric attenuation. ${ }^{(c)}$ The flux density can be obtained as $S_{v}=T_{\mathrm{A}}^{\prime} / \Gamma$ (or equivalently, $S_{v}=\eta_{\mathrm{MB}} T_{\mathrm{MB}} / \Gamma$ ). ${ }^{(d)}$ Central LSR velocity. ${ }^{(e)}$ Fullwidth at half maximum. ${ }^{(f)}$ Velocity-integrated intensity of the line. ${ }^{(g)}$ Channel-to-channel $1 \sigma \mathrm{rms}$ noise of the line-free channels for a velocity resolution of $20 \mathrm{~km} \mathrm{~s}^{-1}$.

not published and it is no longer available in the SEST data archive, preventing us from confirming their results. In addition, Nyman et al. (1992) used the SEST and the Onsala $20 \mathrm{~m}$ Telescope to carry out a survey of CO emission toward a sample of IRAS point sources, including V605 Aql. However, their observations toward V605 Aql reached an rms limit of $\sim 1 \mathrm{Jy}$, which is higher than the peak main-beam temperature reported by Kameswara-Rao et al. (1991), and did not detect any emission.

On the other hand, soon after V4334 Sgr experienced the VLTP, and just before it disappeared from our view at optical wavelengths, Evans et al. (1998) carried out observations with the James Clerk Maxwell Telescope to search for the $\operatorname{CO}(J=$ $2 \rightarrow 1$ ) line toward this object, but no emission was detected above an rms level of $\sim 0.65 \mathrm{Jy}$. To our knowledge, this is the only attempt to search for millimeter/submillimeter CO emission toward V4334 Sgr published in the literature.

Our observations reveal that the molecular emission in the two youngest born-again stars is relatively weak, which explains why previous attempts to detect such emission had failed. On the other hand, the non-detection of molecular emission toward the more evolved objects A30 and A78 is in agreement with the picture of an evolutionary sequence, where the molecules are dissociated since the envelope is photo-ionized by the hot central star. One particularly interesting result from our observations is the clear detection of HCN emission in V4334 Sgr, while the $\mathrm{CO}(J=3 \rightarrow 2)$ line does not appear in its spectrum (lower panel of Fig. 1). Conversely, $\mathrm{CO}(J=3 \rightarrow 2)$ emission is found in V605 Aql, but no $\mathrm{H}^{13} \mathrm{CN}(J=4 \rightarrow 3)$ emission is detected in this source (Fig. 3). It is not clear what the origin of this difference could be, which leads us to wonder whether this could be due to an evolutionary effect in the chemical composition of CSE of these sources, or due to differences between their intrinsic chemical compositions. Particularly, the relatively high content of hydrogen-bearing gas in the recently created CSE of V4334 Sgr indicates that a considerable fraction of hydrogen was blown out instead of being ingested during the VLTP (see Sect. 4.3). Asplund et al. (1997) showed that the surface hydrogen abundance of V4334 Sgr decreased during 1996 and, thus, the molecules may have formed in the earlier ejecta, which still had some hydrogen.

The ${ }^{12} \mathrm{C} /{ }^{13} \mathrm{C}$ abundance ratio in the newly formed CSE of V4334 Sgr has been estimated independently by some authors (Pavlenko et al. 2004; Evans et al. 2006; Worters et al. 2009). In general, they find a low value of $2 \sim 5$, which hints at the occurrence of a VLTP in this source. From our observations, we find that the ratio of the integrated line intensities, $I_{\mathrm{H}^{12} \mathrm{CN}} / I_{\mathrm{H}^{13} \mathrm{CN}}$, is $\approx 3$ (see Table 2). Assuming that both lines arise in the same region and that they are optically thin, this ratio translates into
Table 3. Noise characteristics for non-detected molecular transitions.

\begin{tabular}{llrr}
\hline \hline Molecule & Transition & $\begin{array}{r}\text { Rest frequency } \\
(\mathrm{GHz})\end{array}$ & $\begin{array}{c}\mathrm{rms}^{a} \\
(\mathrm{mJy})\end{array}$ \\
\hline \multicolumn{4}{c}{$\mathrm{V} 4334 \mathrm{Sgr}$} \\
\hline $\mathrm{CO}$ & $J=3 \rightarrow 2$ & 345.79599 & 57 \\
$\mathrm{CO}$ & $J=2 \rightarrow 1$ & 230.53800 & 68 \\
$\mathrm{CO}$ & $J=1 \rightarrow 0$ & 115.27120 & 13 \\
${ }^{13} \mathrm{CO}$ & $J=1 \rightarrow 0$ & 110.20135 & 6 \\
\hline \multicolumn{5}{c}{$\mathrm{V} 605 \mathrm{Aql}$} \\
\hline${ }^{13} \mathrm{CN}$ & $J=4 \rightarrow 3$ & 345.33976 & 29 \\
${ }^{13} \mathrm{CO}$ & $J=3 \rightarrow 2$ & 330.58797 & 86 \\
\hline \multicolumn{5}{c}{$\mathrm{A} 30$} \\
\hline $\mathrm{CO}$ & $J=2 \rightarrow 1$ & 230.53800 & 24 \\
$\mathrm{CO}$ & $J=1 \rightarrow 0$ & 115.27120 & 16 \\
${ }^{13} \mathrm{CO}$ & $J=1 \rightarrow 0$ & 110.20135 & 9 \\
$\mathrm{HCN}$ & $J=1 \rightarrow 0$ & 88.63185 \\
\hline \multicolumn{5}{c}{$\mathrm{A} 78$} \\
\hline $\mathrm{CO}$ & $J=1 \rightarrow 0$ & 115.27120 & 10 \\
${ }^{13} \mathrm{CO}$ & $J=1 \rightarrow 0$ & 110.20135 \\
$\mathrm{HCN}$ & $J=1 \rightarrow 0$ & 6 \\
\hline
\end{tabular}

Notes. ${ }^{(a)}$ Channel-to-channel $1 \sigma$ rms noise of the spectrum smoothed to a velocity resolution of $20 \mathrm{~km} \mathrm{~s}^{-1}$.

the ${ }^{12} \mathrm{C} /{ }^{13} \mathrm{C}$ abundance ratio, which would be in agreement with the values previously found by other authors. Following a similar argument for V605 Aql, using the upper limit for the ${ }^{13} \mathrm{CO}$ emission (Table 3), we obtain a lower limit for the integrated line intensities $I_{{ }^{12}} \mathrm{CO} / I_{13} \mathrm{CO}>4$. Nonetheless, it is important to remark that the data of the $\mathrm{HCN}$ and $\mathrm{H}^{13} \mathrm{CN}$ emission toward V4334 Sgr were taken more than two years apart and, for sources that evolve so rapidly, we have to be cautious when comparing observations that were performed at different epochs.

\subsection{Spatial extent of the molecular emission}

The width of the detected molecular lines, in both sources, show that the emitting material is expanding at relatively large velocities, $v_{\exp } \gtrsim 80 \mathrm{~km} \mathrm{~s}^{-1}$. This clearly indicates that the observed molecular gas is not associated with the fossil planetary nebula, which is expanding at a much lower velocity, $v_{\exp } \sim 20$ $30 \mathrm{~km} \mathrm{~s}^{-1}$, but rather with the material ejected during the bornagain event. If one assumes that the gas expands at a constant velocity, the angular diameter of the emitting region can be estimated as $\theta=v_{\exp } \delta t / D$, where $\delta t$ is the time interval between 
the born-again event and the observation date, and $D$ is the distance to the source. For V605 Aql, the born-again event occurred $\sim 100 \mathrm{yr}$ ago and the distance to the source has been estimated as $4.6 \mathrm{kpc}$ (Clayton et al. 2013). Taking half the value of the linewidth of the $\mathrm{CO}$ emission as the expansion velocity, $v_{\exp }=\Delta v_{\mathrm{CO}} / 2$, the angular diameter of the emitting region is $\sim 0$.' 7 . In the case of V4334 Sgr, the time interval is $20 \mathrm{yr}$; assuming a distance of $3 \mathrm{kpc}$ (Kimeswenger 2002), and calculating the expansion velocity from the average of the linewidths of the HCN emission, $v_{\mathrm{exp}}=\left[\Delta \mathrm{v}_{\mathrm{HCN}(J=4 \rightarrow 3)}+\Delta \mathrm{v}_{\mathrm{HCN}(J=1 \rightarrow 0)}\right] / 2$, the corresponding angular diameter is $\sim 0$. 5 .

From Hubble Space Telescope (HST) images of the [OIII] and [NII] emission, Wesson et al. (2008) measured the diameter of the hydrogen deficient nebula in V605 Aql as being 0.'76. In the case of V4334 Sgr, Hinkle \& Joyce (2014) estimate that the spatial extent of the material traced by emission of He I

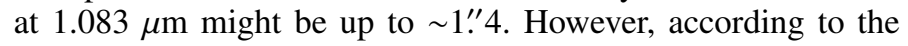
linear relationship for the expansion of the dust shell found by Evans et al. (2006) and Hinkle \& Joyce (2014), the size of the dust shell should be only $\sim 0$." 4 in diameter. From our single dish observations, we cannot tell the true distribution of the molecular emission, but it can be assumed that the molecular emission comes from a region of similar extent as the structures seen at other wavelengths. Interferometric observations with high angular-resolution are necessary to confirm this hypothesis.

\subsection{Column density and rotational temperature of $\mathrm{HCN}$ in V4334 Sgr}

Given that we detected emission of the HCN molecule at two different transitions in V4334 Sgr, we can attempt to estimate the rotational temperature and the column density of this molecule in the following way. Assuming LTE conditions, the rotational temperature, $T_{\text {rot }}$, and total column density, $N$, can be obtained by fitting a linear function in the population diagram (also referred to as rotation diagram) to the observational data using the following equation (Goldsmith \& Langer 1999):

$\ln \frac{N_{u}}{g_{u}}=\ln \frac{N}{Z(T)}-\frac{E_{u}}{k T}$,

where $N_{u}$ is the column density of molecules in the upper state; $g_{u}$ and $E_{u}$ are the statistical weight and energy of the upper level of the transition, respectively; $Z$ is the partition function of the molecule, and $k$ is the Boltzmann constant.

The observed column density of molecules in the upper state, $N_{u}^{\mathrm{obs}}$, is obtained as

$N_{u}^{\mathrm{obs}}=\frac{8 \pi k v^{2} I_{\mathrm{HCN}}}{h c^{3} A_{u l} f_{\mathrm{s}}}\left(\frac{\tau_{v}}{1-\mathrm{e}^{-\tau_{v}}}\right)$,

where $v$ is the rest frequency of the transition, $h$ is the Planck constant, $c$ is the speed of light in vacuum, $A_{u l}$ is the Einstein A-coefficient for spontaneous emission, $I_{\mathrm{HCN}}$ is the velocityintegrated intensity of the line, which is calculated as the product $T_{\text {MB,peak }} \times \Delta v ; f_{\mathrm{s}}$ is the beam-filling factor and $\tau_{v}$ is the optical depth at the peak of the line. The latter two parameters are the main source of uncertainty in determining $N_{u}^{\text {obs }}$.

In principle, the beam filling factor, defined as the fraction of the area of the beam of the telescope filled by the source, can be estimated directly from the angular size of the emitting region which, in the previous subsection, was estimated as $\theta_{\mathrm{s}} \sim 00^{\prime \prime} 5$ in diameter, and assuming a uniform distribution of the gas. However, a clumpy structure of the emitting region would result in a smaller value of the beam-filling factor. Furthermore, since the optical depths of the lines are not well constrained, we start by assuming that the distribution of the gas is uniform, and that the emission of both transitions is optically thin, i.e. $\tau_{v} \ll 1$. Later, we explore how the results change when these assumptions do not hold.

To calculate $N_{u}^{\mathrm{obs}}$ and $E_{u}$ for the two observed lines of the $\mathrm{HCN}$ molecule and to perform the linear fit using using Eq. (1), we used the values of the Einstein A-coefficients, statistical weights, and partition functions from the Cologne Database for Molecular Spectroscopy (Müller et al. 2001, 2005). The fit gives a rotational temperature of $T_{\text {rot }}=13 \pm 1 \mathrm{~K}$, and a total column density of $N_{\mathrm{HCN}}=1.6 \pm 0.1 \times 10^{16} \mathrm{~cm}^{-2}$. Moreover, assuming a distance of $3 \mathrm{kpc}$ and an angular diameter of $0 .{ }^{\prime \prime} 5$ for this source, we derive a total $\mathrm{HCN}$ mass $M_{\mathrm{HCN}}=1.4 \pm 0.1 \times 10^{-7} M_{\odot}$. Evans et al. (2006) analyzed absorption lines of HCN in the infrared and derived values of the temperature and total column density $T_{\text {rot }}=450 \mathrm{~K}$ and $N_{\mathrm{HCN}}=1.4 \times 10^{17} \mathrm{~cm}^{-2}$, respectively. The difference between their values and the ones derived from our observations is likely due to the fact that the gas probed by the infrared observations lies only in the direction between the infrared background and the observer, which is closer to the denser and hotter dust envelope, while we obtain an averaged value over the whole emitting region, which includes cooler and less dense gas.

However, we emphasize that the values obtained from the analysis above should be considered as a first approximation since they are fairly sensitive to the assumptions on the beamfilling factor and optical depth of the lines as follows. From Eq. (1), it can be seen that the rotational temperature is inversely proportional to the absolute value of the slope of the line that best fits the data. Accordingly, the total column density, $N$, is proportional to the value where it crosses the ordinate axis. On the other hand, from Eq. (2), it follows that $N_{u}$ is inversely proportional to the beam-filling factor and linearly proportional to $\tau_{v} /\left(1-\mathrm{e}^{-\tau_{v}}\right)$. Therefore, different beam-filling factors and/or optical depths for each transition cause the values of $N_{u}$ to move upwards and/or downwards in the population diagram, which affects both the slope and the ordinate-crossing point of the bestfitting line. Indeed, as mentioned above, the beam-filling factor could be overestimated if the emitting region has a rather clumpy distribution. In addition, if the transition of higher energy, $\operatorname{HCN}(J=4 \rightarrow 3)$ is preferentially excited closer to the star, the emitting region of this line could be smaller, resulting in a different value of the beam-filling factor for each line. Furthermore, our assumption of optically thin emission leads to an underestimation of the total column density, although the agreement between the $I_{\mathrm{H}^{12} \mathrm{CN}} / I_{\mathrm{H}^{13} \mathrm{CN}}$ and the ${ }^{12} \mathrm{C} /{ }^{13} \mathrm{C}$ ratios suggests that the lines are indeed optically thin. Hence, it is clear that more observations of several transitions at higher angular resolution are necessary to disentangle the physical properties and distribution of the molecular gas in this source.

\section{Conclusions}

Using the APEX and IRAM $30 \mathrm{~m}$ telescopes, we carried out a search for molecular emission toward four sources that are known to have experienced a VLTP and detected emission from V4334 Sgr and V605 Aql. This is the first time that millimeter/sub-millimeter molecular emission is detected in born-again stars. While the $\mathrm{HCN}$ and $\mathrm{H}^{13} \mathrm{CN}$ emission is strong in V4334 Sgr, it does not appear in the spectrum of V605 Aql with the same rms noise level. Conversely, CO emission was detected in V605 Aql but not in V4334 Sgr. This result might point to differences in the chemical composition of these 
sources, or to a chemical evolution in their circumstellar envelope. On the other hand, the non-detection of molecular emission in the other two, more evolved, born-again stars (A30 and A78) is in agreement with an evolutionary scenario, where the molecules are destroyed as the central star re-heats and ionizes the CSE. For V4334 Sgr, under the assumptions of LTE and optically thin emission, we derived a rotational temperature for the molecular gas of $T_{\text {rot }}=13 \pm 1 \mathrm{~K}$, and a total column density of $N_{\mathrm{HCN}}=1.6 \pm 0.1 \times 10^{16} \mathrm{~cm}^{-2}$. Considering a distance of $3 \mathrm{kpc}$ and a size of 0. . 5 , the total HCN mass in V4334 Sgr is $M_{\mathrm{HCN}}=1.4 \pm 0.1 \times 10^{-7} M_{\odot}$. The ratio of the integrated line intensities, $I_{\mathrm{H}^{12} \mathrm{CN}} / I_{\mathrm{H}^{13} \mathrm{CN}}$, is $\approx 3$ in V4334 Sgr. Assuming that both lines are optically thin, this value represents the ${ }^{12} \mathrm{C} /{ }^{13} \mathrm{C}$ abundance ratio, which would be in agreement with previous estimations from infrared observations. Under similar assumptions for V605 Aql, we find a lower limit of the abundance ratio ${ }^{12} \mathrm{C} /{ }^{13} \mathrm{C}>4$. Future observations at high angular resolution will reveal the distribution and kinematics of the gas ejected by these born-again stars. This will be an important step toward understanding the formation of asymmetries in the CSE of evolved stars.

Acknowledgements. The authors thank Karl Torstensson for his valuable help with the observations at the APEX site and to Magnus Persson for helpful discussions. The authors also thank the anonymous referee for constructive comments and suggestions that helped to improve the manuscript. The APEX dat were obtained via Onsala Space Observatory observing time and are archived with the program identification codes 092.F-9330 and 097.F-9341. D.T. and W.V. acknowledge support from ERC consolidator grant 614264. A.A.Z. acknowledges funding from the UK STFC research council. Á.S.-M. thanks the Deutsche Forschungsgemeinschaft (DFG) for funding support via the collaborative research grant SFB 956, project A6. S.P.T.M. thanks the Spanish MINECO for funding support from grants AYA2012-32032, CSD2009-00038, FIS2012 32096, and ERC under ERC-2013-SyG, G. A. 610256 NANOCOSMOS

\section{References}

Asplund, M., Gustafsson, B., Lambert, D. L., \& Kameswara-Rao, N. 1997, A\&A, 321, L17

Balick, B., \& Frank, A. 2002, ARA\&A, 40, 439

Benetti, S., Duerbeck, H. W., Seitter, W. C., Harrison, T., \& Hoff, W. 1996 , IAU Circ., 6325

Borkowski, K. J., Harrington, J. P., Tsvetanov, Z., \& Clegg, R. E. S. 1993, ApJ, 415, L47

Borkowski, K. J., Harrington, J. P., Blair, W. P., \& Bregman, J. D. 1994, ApJ, 435, 722

Chesneau, O., Clayton, G. C., Lykou, F., et al. 2009, A\&A, 493, L17
Chu, Y.-H., Chang, T. H., \& Conway, G. M. 1997, ApJ, 482, 891 Clayton, G. C., \& De Marco, O. 1997, AJ, 114, 2679

Clayton, G. C., Kerber, F., Pirzkal, N., et al. 2006, ApJ, 646, L69 Clayton, G. C., Bond, H. E., Long, L. A., et al. 2013, ApJ, 771, 130

De Marco, O. 2014, in Asymmetrical Planetary Nebulae VI Conf. Proc., 4-8 November 2013, eds. C. Morisset, G. Delgado-Inglada, \& S. Torres-Peimbert, 122

Duerbeck, H. W., Pollacco, D., Verbunt, F., et al. 1996, IAU Circ., 6328, 1

Duerbeck, H. W., Benetti, S., Gautschy, A., et al. 1997, AJ, 114, 1657

Duerbeck, H. W., Liller, W., Sterken, C., et al. 2000, AJ, 119, 2360

Evans, A., Eyres, S. P. S., Naylor, T., \& Salama, A. 1998, A\&A, 335, 292

Evans, A., Tyne, V. H., van Loon, J. T., et al. 2006, MNRAS, 373, L75

Eyres, S. P. S., Evans, A., Geballe, T. R., Salama, A., \& Smalley, B. 1998 MNRAS, 298, L37

Fang, X., Guerrero, M. A., Marquez-Lugo, R. A., et al. 2014, ApJ, 797, 100

Goldsmith, P. F., \& Langer, W. D. 1999, ApJ, 517, 209

Guerrero, M. A., \& Manchado, A. 1996, ApJ, 472, 711

Guerrero, M. A., Ruiz, N., Hamann, W.-R., et al. 2012, ApJ, 755, 129

Hajduk, M., Zijlstra, A. A., Herwig, F., et al. 2005, Science, 308, 231

Herwig, F. 2002, Ap\&SS, 279, 103

Hinkle, K. H., \& Joyce, R. R. 2014, ApJ, 785, 146

Hinkle, K. H., Lebzelter, T., Joyce, R. R., et al. 2008, A\&A, 479, 817

Iben, Jr., I. 1984, ApJ, 277, 333

Jacoby, G. H. 1979, PASP, 91, 754

Kameswara-Rao, N., Nyman, L. A., Nandy, K., \& Houziaux, L. 1991, in The Infrared Spectral Region of Stars, eds. C. Jaschek, \& Y. Andrillat (Cambridge University Press), 361

Kimeswenger, S. 2002, Ap\&SS, 279, 79

Kimeswenger, S., Kerber, F., \& Weinberger, R. 1998, MNRAS, 296, 614

Kwok, S., Purton, C. R., \& Fitzgerald, P. M. 1978, ApJ, 219, L125

Miller Bertolami, M. M., Althaus, L. G., Serenelli, A. M., \& Panei, J. A. 2006, A\&A, 449, 313

Müller, H. S. P., Thorwirth, S., Roth, D. A., \& Winnewisser, G. 2001, A\&A, 370 , L49

Müller, H. S. P., Schlöder, F., Stutzki, J., \& Winnewisser, G. 2005, J. Mol. Struct. 742,215

Nakano, S., Sakurai, Y., Hazen, M., et al. 1996, IAU Circ., 6322,

Nyman, L.-A., Booth, R. S., Carlstrom, U., et al. 1992, A\&AS, 93, 121

Pavlenko, Y. V., Geballe, T. R., Evans, A., et al. 2004, A\&A, 417, L39

Phillips, J. P., \& Ramos-Larios, G. 2007, AJ, 133, 347

Pollacco, D. L., Lawson, W. A., Clegg, R. E. S., \& Hill, P. W. 1992, MNRAS, 257, 33

Seitter, W. C. 1987, The Messenger, 50, 14

Toalá, J. A., Guerrero, M. A., Todt, H., et al. 2015, ApJ, 799, 67

van Hoof, P. A. M., Hajduk, M., Zijlstra, A. A., et al. 2007, A\&A, 471, L9

Wesson, R., Barlow, M. J., Liu, X.-W., et al. 2008, MNRAS, 383, 1639

Wilson, T. L., Rohlfs, K., \& Hüttemeister, S. 2013, Tools of Radio Astronomy, 6th edn., Astron. Astrophys. Lib. (Berlin, Heidelberg: Springer), 173

Wolf, M. 1920, Astron. Nachr., 211, 119

Woodward, P. R., Herwig, F., \& Lin, P.-H. 2015, ApJ, 798, 49

Worters, H. L., Rushton, M. T., Eyres, S. P. S., Geballe, T. R., \& Evans, A. 2009, MNRAS, 393, 108

Zijlstra, A. A. 2002, Ap\&SS, 279, 171 